\title{
Will designated patient navigators fix the problem? Oncology nursing in transition
}

\section{by Sally Thorne and Tracy Truant}

\begin{abstract}
With increasing concern for equity and access across the cancer care system, we have seen expanding enthusiasm for various forms of designated patient "navigators" to facilitate coordination. While the intention is laudable, many of the popular implementation strategies risk accentuating strain upon the system and further complicating the coordination problem. These authors claim the motivation underlying the navigator movement can be reframed as an emerging recognition of the value of nursing work when it is optimally positioned to support patients, as they experience the cancer care system. This paper calls on Canadian oncology nurses to critically challenge navigation strategies, and adopt only those consistent with the significant reforms required to ensure a cancer care system so effective that external navigators are no longer necessary.
\end{abstract}

Large numbers of patients living with, through and beyond cancer in Canada clearly and consistently have described their experience of the Canadian cancer care system as fragmented, sometimes inaccessible, and "like a maze" (Canadian Strategy for Cancer Control, 2002). Associated with these discontinuities of care are high levels of psychosocial distress related to unmet information needs, inadequate support, or communication breakdown, all of which negatively affect quality of life and increase the burden of suffering throughout the cancer experience (Bultz \& Carlson, 2006; Bultz \& Holland, 2006; Doll et al., 2003; Fillion et al., 2006; Fitch, Cook, \& Plante, 2008). A variety of solutions have been proposed to address these issues, including patient navigation models that propose to improve the cancer experience for patients and families across the cancer journey.

\section{Background}

As enthusiasm for understanding the "cancer journey" has increasingly found its rightful place in the policy agenda, there has been a flurry of interest in the concept of "patient navigators" to ensure equitable and efficient access to cancer services and to bridge gaps in care across the cancer journey (Fitch, Cook, et al., 2008). The concept of patient navigators in cancer care originated almost two decades ago in Harlem hospitals, as a mechanism to correct what were considered unacceptable disparities in cancer mortality between minority African-American and other population groups (Darnell, 2007). The idea underlying the introduction of patient navigators was to identify populations with "excess cancer mortality" and to provide patients within those populations "personal assistance in eliminating any barriers to patients obtaining timely and adequate diagnosis and treatment" (Freeman, 2004, p. 76). As such, the patient navigator concept represented a passionate attempt to eradicate what were clearly recognized as unacceptable cultural, economic, societal and logistical impediments to timely diagnosis and appropriate treatment, so that all patients might benefit from the best available science and health care.

Across the United States (U.S.), where access to health services remains decidedly uneven, the patient navigator concept advanced by Freeman was taken up by various reformers, and has eventually taken hold within the national policy agenda. In 2002, the National Cancer Institute began funding research programs directed toward this approach, as part of its Cancer Disparities Research Partnership Program (Dohan \& Schrag, 2005), and had authorized the appropriation of significant funding to support considerable expansion of similar initiatives (Davenport-Ellis, 2007). Dr. Freeman, the originator of the patient navigation concept, was enlisted to lead the charge nationally. From this initiative, a number of projects have been launched and, as a result, the literature is now beginning to reveal an expanding body of knowledge related to the cost, efficacy and outcomes of various patient navigation models and approaches.

\section{The Canadian context}

Although the fundamental disparities inherent in the American health care system are not the primary challenge in our Canadian context, a shared and strong multicultural sensibility has led many Canadian researchers to document issues related to group differences that we continue to experience here at home. Thus, a deep concern for the "politics of difference" (Browne \& Tarlier, 2008) has led many Canadian health system reform advocates to champion the idea of patient navigators, as the optimal solution to ensuring support for the most vulnerable of patients. Since all systems recognize that there are some individuals whose needs are sufficiently complex that providing care will disproportionately draw on system time and resources, the idea of navigators who might personalize attention to their needs has an obvious appeal. However, because Canada's health care system is built on the premise of equal access for a highly diverse population within a publicly funded system, deciding which particular diversities justify which kinds of special rights and privileges within the system is a complex and potentially divisive policy problem.

Around the same time the National Institutes of Health (NIH) in the U.S. had begun to establish funding for navigation research in 2002, federal funding was secured in Canada to develop a Canadian Strategy for Cancer Control (CSCC), whose aims included ensuring equitable access to services and resources for all Canadians at risk/living with cancer. The CSCC, which eventually evolved into the Canadian Partnership Against Cancer (CPAC), appointed various "action groups" to create a strategy toward meeting the needs of Canadians at risk for/living with cancer. The ReBalance Focus Action Group (now called the Cancer Journey Action Group) was established to shift the focus of cancer care beyond the diseasefocused issues associated with diagnosis and treatment, so that it would better meet the informational, physical, psychosocial, emotional, spiritual, nutritional and practical needs of patients and their

\section{About the authors}

Sally Thorne, RN, PhD, FCAHS, Professor and Director, UBC School of Nursing, T201-2211 Wesbrook Mall, Vancouver, BC V6T 2B5. Phone: 604-822-7748; Fax: 604-822-7423; E-mail: sally.thorne@nursing.ubc.ca

Tracy Truant, RN, MSN, Regional Professional Practice Leader, Nursing, BC Cancer Agency, Vancouver, BC. 
families across the cancer care journey from pre-diagnosis through to survivorship or palliative care and bereavement (Fitch, 2008). In the context of these national action group discussions, cancer patient navigation was identified as one potential strategy with which to meet the supportive care needs of patients and families across a fragmented, complex and sometimes inaccessible cancer care system (Fitch, Cook, et al., 2008).

In this paper, we draw upon insights gleaned from both research and practice experience to reflect critically on the implications for both nurses and patients of uncritical endorsement of the current trend toward new systems of patient navigation. One of us comes from the perspective of longstanding engagement in consumer-based research involving persons seeking care for cancer within our Canadian health care system. The other brings the perspective of a longstanding clinician and leader of oncology nursing practice systems. We use the intersection of these distinct angles of vision to illuminate an alternative conceptualization of the fragmentation problem, critically reflect upon the current patient navigator agenda, and propose alternative strategic directions for oncology nursing within the Canadian cancer care context.

\section{The patient perspective}

Although chronic disease and cancer have historically been considered fundamentally distinct clinical specialties in nursing, it is increasingly recognized that they are prevalent in the same populations and share many common features relevant to prevention, supportive care and survivorship. When patients with cancer and chronic disease are asked how they experience the health care system and about their encounters with the people within it, it is perhaps not surprising that they describe rather similar patterns of what constitute essential system navigation problems. Further, a remarkably similar set of barriers, problems and challenges is reported not only on the basis of studies of populations who are vulnerable by virtue of sociocultural or economic disadvantage, but rather across the full patient spectrum (Decter \& Grosso, 2006). Thus, notwithstanding the very real additional challenges experienced by certain linguistic or ethnic groups, for example, the challenge of negotiating health care seems a daunting one for all Canadians, and a matter deserving particular attention within the context of the patient cancer journey.

A recent program of qualitative research into the patient experience of communication in cancer care by the first author (reported elsewhere) provided an opportunity to review and reflect on the stories of 260 British Columbian cancer patients representing a wide range of tumour sites, treatment modalities, and demographic features. Thematic patterns in relation to the patient navigation issue arising from this data set were discussed in depth with the second author, whose experiential involvement in cancer care process systems over this same period of time afforded the opportunity for a provider perspective of the same period in time. Reflective analysis of the study findings in the context of perceptions deriving from experiential practice allowed us to expand and elaborate upon trends within the research and explore additional interpretations. The ideas presented here evolved as a result of that analytic dialogue.

\section{What needs to be navigated?}

\section{The primary care gap}

While it is well recognized that a coordinated cancer care system inherently relies upon high-quality primary care, not all Canadians have a family doctor, and those who do may have considerable difficulties with access (Decter \& Grosso, 2006). Although system reforms are underway to expand the primary care system beyond the conventional family physician, who is often in solo practice, to integrated and interprofessional community-based networks (Canadian Institute for Health Information, 2008), the reform process has been protracted and painful (Murray et al., 2008). Where effective coordinated primary care exists, cancer patients are supported in accessing prevention, screening, early detection and accessing referral to specialty systems. However, far too often, primary care exists in the context of walkin-clinics, whose orientation is toward sporadic urgent care episodes rather than patient follow-up and continuity of care. Emergency departments have become the default primary care site for those who fall between the cracks. If we understand that the current organization of our entire system revolves around the assumption of an appropriately functioning primary care system, then we begin to appreciate how pervasive and extensive the navigation problem really is, and that approaches to addressing it within the cancer context have to begin long before a patient receives the cancer diagnosis.

\section{Fragmentation of specialization}

Once fully ensconced in the specialty cancer care system, patients continue to experience challenges with regard to continuity and collaboration among their cancer care team and specialists. It is increasingly acknowledged across all disciplines that what has driven conventional science in the direction of increasing specialization has concurrently created major gaps in our capacity to see the larger picture and understand issues at a system level (Dorr et al., 2006). While specialization has undoubtedly advantaged our capacity to answer certain questions, it has also created new problems. Because the holders of expertise within a specialty are inherently invested in advancing the primacy of that specialty within the system, and because society's dependence upon the services associated with expertise can be applied toward social persuasion or political influence, power struggles and turf wars have been allowed to flourish and to influence policy judgments in health system design and redesign (McKay \& Crippen, 2008; McMurtry \& Bultz, 2005).

Although there is an increasing trend toward recognizing that complex adaptive systems such as health care require coordination and "big system" thinking at policy tables, the Canadian health care system continues to suffer from a historic reliance upon reductionist thinking produced by specialists within particular organ systems, treatment modalities, or services. While we commonly claim to value care coordination and patient outcomes, most members of the typical health care team are functionally accountable only for their own distinct piece within the service constellation, and very few have a formal mandate to even notice, let alone influence, what happens to patients across services and sectors. And, yet, we know that the care coordination problem is persistent and greatly detrimental to patient experience and outcomes.

\section{The cancer care "team"}

Even within specialized interprofessional cancer care teams, lack of collaboration and fragmentation of care are common problems, leaving patients to find their own way through the maze (Reid Ponte, Gross, Winer, Connaughton, \& Hassinger, 2007). In order for patients to experience continuity of care across multiple care providers within the interprofessional team, often located across various settings, providers must communicate, trust one another, understand and respect one another's role and scope of practice, and maintain a collaborative sense of responsibility to their patients, as they guide them through the cancer journey (Fleissig, Jenkins, Catt, \& Fallowfield, 2006). Further, the team needs to rally together around a patient-centred approach to care, which includes an intentional effort that consciously adopts the person's perspective about what matters and attends to the whole person (McMurtry 
\& Bultz, 2005; Willard \& Luker, 2005). In this way, effective interprofessional teams can optimally work together to close the gaps for patients across the cancer journey, thereby decreasing the need for an external navigator.

\section{Other blind spots within the system}

Our Canadian health care system, as with health systems globally, grew out of a time when curative physician services and hospital care were the exclusive focus of public policy regarding health care (Decter \& Grosso, 2006). Over time, as knowledge has advanced, it has become apparent that health is an inherent feature of the societies in which we live, and that health promotion, prevention and disease management are far more influential on the overall health of society than is intervention toward cure (McKenna \& Zohrabian, 2009). Despite such advances, many elements of health service structure remain firmly aligned with the assumptions upon which health service delivery was originally established. For example: our policies overemphasize service entrance restriction to the point that it is counterproductive to appropriate access; we often consider knowledge to be the prerogative of the professional and not the patient; we organize the vast majority of care around the assumption that the physician is the most appropriate team captain; and we favour reactivity over pro-activity in relation to service provision for predictable patient and population problems (Epping-Jordan, Pruitt, Bengoa, \& Wagner, 2004). While numerous individual health care professionals and even groups of professionals sense the fundamental flaws in these system elements and strive to overcome them in their everyday practice, their efforts are often thwarted by an overall structural and philosophical resistance to change that seems endemic to health care systems (Fleissig et al., 2006).

\section{The communication challenge}

Somewhere within the context of these rather massive structural and attitudinal features of the health care system are the individual workers and care providers with whom each cancer patient will interact, either directly or indirectly. For most patients, these people become the "face" of the health care system, and the human manifestations of its capacity and willingness to help them during their time of need (Thorne, Kuo, et al., 2005). Although each of us recognizes how fundamental communication is to the human condition, the ubiquitousness of communication makes it quite difficult to address formally, as a clinical competency or a service system attribute. Thus, while we might generally appreciate that good communication adds value, we have very little hard evidence that any one communication approach is universally preferable to any other, or that any specific instance of communication in which we engage has any correlation to meaningful clinical outcomes (Thorne, Hislop, Armstrong, \& Oglov, 2008). Thus, until recently, communication featured primarily as a "motherhood" abstraction in organizational values statements and vague commitments in terms of practice standards, with very little explicit attention in professional or system performance assessment. However, as advances in systems analysis bring the role of communication in coordination, patient safety, and outcomes increasingly into focus, there seems a newfound interest in paying attention to what patients have been telling us all along-that communication really does matter (Thorne, Bultz, Baile, \& SCRN Communication Team, 2005).

\section{Where should we be going?}

Serious attention to what patients and advocacy groups are telling us about the disjunctures within our system forces us to recognize that we operate within a cancer care system that was not designed with a patient-centred approach or the patient journey in mind. In the context of these recurring coordination problems, it is, perhaps, understandable that designated patient navigators have quickly captured widespread attention as a viable solution. However, on the basis of our analysis of the situation gleaned from extensive patient interviewing and from frontline practice, we are convinced that it is important for Canadian oncology nurses to reflect carefully and critically on this trend and to consider what it might mean for us, for the system and, ultimately, for patients. Here, we raise concerns related to the context in which navigation models have arisen, the potential unintended consequences of some of the various models, and the fundamental problem inherent in delegating navigation to a designated set of additional health care workers.

\section{Critiquing the navigation agenda}

Without question, navigation seems to have become the presenting symptom of a system with inherent ideological, cultural and organizational problems. While few would seriously question the potential benefit of designated navigation for certain vulnerable populations (Wells et al., 2008), the specific circumstances that have created the navigation problem in the Canadian health care system are not the same as those from which the patient navigator movement arose in the U.S. Instead of reflecting systemic racism that disadvantaged one population over another, our coordination issues, for the most part, derive from the embedded attitudes and ideologies that entrench old ways of conceptualizing issues of health care access, scope and responsibility (World Health Organization, 2002). Thus, if we recognize that these factors affect the broad population rather than a few particularly disadvantaged individuals, then our equal access commitment would suggest that if navigators are needed to manage within the system, they ought to be needed by patients across the full spectrum of ethnic and social groupings.

The recent upsurge of enthusiasm by both planners and administrators in aligning with consumer groups to promote a navigation agenda (Doll et al., 2003; Fischer, Sauaia, \& Kutner, 2007; Freund et al., 2008; Nguyen \& Kagawa-Singer, 2008; Schwaderer \& Itano, 2007; Seek \& Hogle, 2007) seems to us a superficial remedy to try to correct a significant dysfunction within the system. While it may be completely understandable that anxious patients would welcome the idea of having someone to guide them through a broken system, we would argue that covering up a mortal wound only delays determination of the root cause for the bleeding. The introduction of designated patient navigators can, therefore, be seen as a well intended, but potentially quite misguided attempt to "BandAid" what is a much larger and more fundamental system integrity problem (Sofaer, 2009; Wells et al., 2008). What is badly needed is a serious public sector commitment to directional change in how we do business in health care (Skrutkowski et al., 2008). Therefore, we owe it to Canadians to try to address the fundamental causes of these current disjunctures, rather than simply attempting to splint them with the quick introduction of a new category of health care worker.

When we insert designated "patient navigators" into the system, while we may temporarily ameliorate certain problems, we also inadvertently contribute to a much more worrisome set of difficulties pertaining to human resource distribution, relational strain within the health care team, and system accountability. For example, where designated navigators are drawn away from the general cancer nursing workforce, we place additional strain upon this already scarce resource, paradoxically increasing the need for external patient navigation. Where non-professionals or lay navigators are injected into the system, as patient advocates, that role poses a significant potential for adversarial interactions, including a predictable tendency to blame individuals and services rather than understanding systemic factors. We may, therefore, increase the level of distrust patients have for their professional health care providers and increase the frequency of litigious responses 
where issues arise. This would be of particular concern where lay navigation extends beyond supporting the specific challenges faced by particularly disadvantaged patients in collaboration with a nursing team and becomes more broadly positioned. Thus, while nurse navigators certainly have the knowledge and skill to meet patients' immediate navigation needs, and while informed lay persons may bring experiential knowledge of how to "work" systems, both models may place the overall system at increased risk for exacerbating the problems that created the need for navigation in the first place.

Another aspect of this problem is the fundamental assumption that designated navigators are responding to systems rather than becoming an inherent part of them. In the majority of contemporary models being advanced in the literature, designated "patient navigators" exist as a resource to patients, as they find their way through systems, and not as an integral member of the health care team (Sofaer, 2009). In essence, they operate like the kindly tour bus driver, who may ensure you are going in the right direction and disembark at the right stop, and who may even help download your luggage, but cannot accompany you to your final destination. The separation of responsibility for navigation from the core function of the interprofessional health care team seems to take us further from resolving the systemic issues that fragment care.

Thus, the navigation agenda, as it is currently being conceptualized and enacted, seems to absolve health professionals, the health care team, and system administrators from responsibility for causing, perpetuating and, indeed, resolving the essential coordination problem. In advocating the introduction of a new "cottage industry" in the form of designated specialist whose exclusive role is to help patients through the system, it creates an additional layer of activity that, itself, will require coordination and a group of workers who may (if one takes a cynical perspective, and our patients often do) become invested in ensuring that the system remains insufficiently well coordinated to justify the continuation of their services.

In contrast, what we really need is a health care team within which the navigation capacity is self-generating and fully integrated (Fitch, 2008; Fitch, Porter, \& Page, 2008). While it makes obvious sense for one member of a well-functioning multidisciplinary health care team to serve as the primary coordinator for the inherent complexities associated with information, management, support and follow-through for each person who enters the system as an actual or potential patient, the imperative of patient navigation ought still to be a shared value across all team members. If we believe that comprehensive, coordinated care optimizes patient outcomes, then navigation issues must be considered as fundamentally important as diagnostic, clinical management or support service issues within our multidisciplinary health care team deliberations and activities. Thus, it seems most appropriate that nursing assume a leading role in taking up the general idea of patient navigation, understanding what motivates it and what it is designed to solve, so as to create the kinds of service structures and processes that will integrate the ideal into the everyday enactment of professional practice and system design.

\section{Considering what this means for nursing}

Open and enthusiastic critical deconstruction of how the health care system has evolved into what we now experience is a useful and enlightening beginning. Once we recognize the ways in which our political, professional and scientific history are a powerful determinant of our current assumptions and actions, we can better position ourselves to become part of what is needed to rebuild a system that prioritizes issues like patient experience and continuity of care. With an understanding of how we got here, we are better prepared to become major contributors to meaningful solutions and, indeed, failure to act makes us complicit in sustaining those barriers to optimal patient care.
While nurses are often quick to criticize other health professions for their narrow specialty focus, our profession also reflects many of the same shortcomings. For example, relatively few nurses self-identify as having a particular interest or expertise in primary care. However, if primary care is the fundamental coordinating structure that explains how and why patients gain access to our services, we must make it a collective disciplinary priority to be actively engaged in solving primary care problems. Further, even within specialty care areas such as oncology, nursing sub-specialization into areas such as systemic therapy, radiation therapy, and palliative care has the potential to intensify the fragmentation problem.

It is essential that we find ways to quickly understand and seamlessly share the patient's story, from the patient's perspective, across disciplines, specialties and settings. It also goes without saying that we need to work toward better communication between parts of the system (including services, professions, and, of course, the patient) and to ensure an appropriate system of transitions between team players. As environments in which the human diversity of the inhabitants is a celebrated strength, health care systems should no longer tolerate privileging the needs of certain providers over others and over the needs of patients. We know that it is impossible to build an effective baseball team from a few star pitchers and a group of batboys. What is needed is a well-differentiated group of skilled players, each understanding and enacting his or her unique role, albeit knowing and caring enough about the roles of others to cover all bases, as needed. If the team doesn't click, no matter how many stars are on board, it doesn't succeed. In health care, as in baseball, we need a deep-rooted and unshakeable shared commitment to interprofessional and multidisciplinary team playing.

Beyond system communication, we also have a key role to play in advancing expectations regarding interpersonal communication. Communication is the context in which information is conveyed, expectations are guided, and compassion is expressed. It is well established that interpersonal communication gaps or inadequacies between professionals and with patients are at the root of the majority of health system failures and errors (Epstein \& Street, 2007). Recognizing the centrality of communication, we must work to ensure we behave as if we recognize its importance. While we would not hesitate to act upon a medication error, we tend toward silence or withdrawal in the context of a communication error, even though its impact may be just as devastating for the patient. We must never forget that it is well within our power to enact and demand an expected standard of communication across all health care interactions. Indeed, these abiding values for teamwork and communication ought to apply to everyone privileged to hold a role within the cancer care system. Whether one is the parking attendant, the CEO, or any provider of services in between, we all have an essential role to play in facilitating patient-centred care along the cancer patient journey.

\section{Proposing strategic alternatives}

The complex skill set associated with understanding diagnostic and care pathways, interpreting concepts and information across disciplinary cultures and finding ways to be optimally positioned for vigilant stewardship of the patient experience has long been the conventional role of the nurse within the hospital sector. While the $24 / 7$ work world of hospital care situates nursing well to enact these roles in the inpatient context, a significant proportion of cancer care takes place in ambulatory settings. However, in the ambulatory context, in particular, the work of oncology nurses is often structured in a manner designed to serve oncologists or treatment services, rather than to coordinate patients' needs across their cancer journey. 
Although many groups of nurses have recognized this essential problem and made great strides toward addressing it, some jurisdictions in Canada have demonstrated significant resistance to the reorganization of nursing within ambulatory care, in the mistaken belief that supporting physicians and treatments prevents error. However, once we recognize the enormity of the threats to patient safety occasioned by a failure to communicate and coordinate, it makes sense to try to strengthen, not reduce, the capacity of nursing through positioning in such a manner that navigation becomes possible. This requires not only recognition of the value of that role within the system, but also policies and practices that ensure direct patient access, permit time distribution to be determined by patient rather than system need, and ensure continuity across services and sectors (Schroeder, Trehearne, \& Ward, 2000).

Where it has been recognized that supporting oncology nurses to organize in ways that facilitate these functions, such as with the infirmière pivot en oncologie (IPO) initiative in Quebec, there is an emerging body of evidence suggesting that systems can become more efficient and effective and patients can fare better in understanding their care and transitioning safely through it (Chasen \& Dippenaar, 2008; De Serres \& Beauchesne, 2000; Fillion et al., 2006; Mick, 2008; Skrutkowski et al., 2008). Despite the very real complexities associated with this kind of research on the impact of nursing roles on patient outcomes, it is important that nursing continues to work toward evaluation of system and patient impacts of various models of navigational support.

\section{Conclusions}

There are increasing signs that we are entering a time in which it becomes possible to create a coordinated voice for change. Advances in our capacity to extract, analyze and interpret population data are producing indisputable evidence that meaningful system change requires the full spectrum of engagement from prevention through specialty practice, from patients and the general public to professional and governmental stakeholders. Further, such change will require breaking down the fragmenting effects of specialization and learning to conceptualize complex problems at more global levels. In Canada, for example, we are seeing such innovations as collaboration across various chronic disease and cancer organizations to join forces toward a strategic national agenda for health promotion and disease prevention. Just as the wider society is beginning to grab hold of an appreciation that environmental change is the kind of issue that requires all of us to make a difference, there is a dawning recognition that we each can play a meaningful part in steering health care toward the kind of effective and efficient system that our society requires. We may even discover unexpected opportunities with the added catalyst that an economic recession provides to "reset" how we deliver care and articulate a more realistic priority framework. In this context, oncology nurses must individually and collectively appreciate that they are integral agents of system quality and not mere bystanders in a system controlled by others.

As a myriad of "system fixes" arise within our various jurisdictions across the country, Canada's oncology nurses must ensure that they possess solid mechanisms with which to critically interpret these trends and mobilize collective actions to best possible solutions. While our profession may have a history of assuming a "victim mentality" in relation to the order and organization of health care (Kitson, 2004), now seems the time for renewed self-con-

\section{References}

Browne, A.J., \& Tarlier, D.S. (2008). Examining the potential of nurse practitioners from a critical social justice perspective. Nursing Inquiry, 15(2), 83-93. fidence and for strategic action. The enthusiasm for investment in navigation actually confirms the inherent value of what we generally consider the "invisible" work of nursing, something we have championed long and hard to justify within the planning agenda. Patients and those responsible for planning and administering cancer care systems may not always understand that strengthening the nursing system is the answer, and it will be our job to ensure that they make the associative link between what is needed and what we are capable of delivering. We need to reframe their perspective so they appreciate that a system in need of navigation is one in which nurses are not yet being optimally deployed.

That coordination is a fundamental problem in health care, and one that can have a profound impact upon patients and outcomes, is a familiar insight for nurses. We have been painfully aware of these issues over the decades, and experienced considerable frustration in our attempts to address them. However, we seem to have before us a new opportunity derived from relatively recent evidence and also new high-level (national and international) policy support for system improvements. The kinds of care coordination, interprofessional teamwork, and shared care changes in health service delivery that many global health policy advocates are now suggesting fit nicely with the kinds of trends that nursing has consistently proposed.

Nurses must always be sensitive to the fine line between advocating "for nursing" and advocating "for patients" from a distinctly nursing perspective. In this instance, the goals of our advocacy are not at all about advancing the profession; rather they arise out of an abiding conviction informed by the unique and distinctive perspective that comes from being a nurse in close proximity to the way patients experience both their cancer and the health care services to which they have access. We know that, where nursing is well positioned within an informed care coordination capacity, and is appropriately situated to interpret the myriad of patient concerns within the interprofessional team context, nurses are the ideal team member to ensure that no patient gets lost in the shuffle. In other words, the stronger the system of nursing, the less there will be any need for dedicated or external "navigators."

From our perspective, the collective voice of nursing must be raised at this critical juncture in our history toward ensuring that the organization of the workforce across the cancer care system fully optimizes nursing's individualization and coordination functions. The old model of organizing oncology nursing as "handmaiden to the specialist" seriously underutilizes the sophisticated competencies that nursing brings to the system, and perpetuates the fragmentation that so dangerously compromises patient outcomes. Our collective objective at this time in our history must be to ensure that all cancer patients and family members have consistent and timely direct access to nurses who are positioned in such a manner that they can come to know patients as unique individuals within their own distinct contexts, skillfully coordinate their transitions, and serve as their engaged and integrated knowledge brokers across the full spectrum of clinicians, teams, and services within the cancer care system.

Thus, "patient navigators" will not solve the problem. In fact, our goal should be a cancer care system so effective that designated patient navigators are no longer needed. Strengthening the capacity of nursing through creative role reconfiguration and responsibility enhancement within the fully functioning interprofessional care team is the key to achieving that goal.

Bultz, B.D., \& Carlson, L.E. (2006). Emotional distress: The sixth vital sign-Future directions in cancer care. Psycho-Oncology, 15, 93-95. 
Bultz, B.D., \& Holland, J.C. (2006). Emotional distress in patients with cancer: The sixth vital sign. Community Oncology, 3(4), 15-18.

Canadian Institute for Health Information. (2008). Health care in Canada 2008. Ottawa: CIHI.

Canadian Strategy for Cancer Control. (2002). Supportive care/cancer rehabilitation workgroup: Final report. Ottawa, ON: Author.

Chasen, M.R., \& Dippenaar, A.P. (2008). Cancer nutrition and rehabilitation: Its time has come! Current Oncology, 15(3), 117-122.

Darnell, J. (2007). Patient navigation: A call to action. Social Work, 52(1), 81-84.

Davenport-Ellis, N. (2007). Access to healthcare: Using data from a non-profit advocacy practice to drive policy change. In J.A.L. Earp, E.A. French, \& M.B. Gilkey (Eds.), Patient advocacy for health care quality: Strategies for achieving patient-centered care (pp. 419-444). Sudbury, MA: Jones and Bartlett.

De Serres, M., \& Beauchesne, N. (2000). L'intervenant pivot en oncologie, un rôle d'évaluation, d'information et de soutien pour le mieux-être des personnes atteintes de cancer [The pivot nurse in oncology, an evaluation, information, and support role for the well-being of people with cancer]. Quebec: Gouvernement du Quebec.

Decter, M., \& Grosso, F. (2006). Navigating Canada's health care: A user guide to getting the care you need. Toronto: Penguin.

Dohan, D., \& Schrag, D. (2005). Using navigators to improve care of underserved patients: Current practices and approaches. Cancer, 104(4), 848-855.

Doll, R.D., Stephen, J., Barroetavena, M.C., Linden, W., Poole, G., \& Habra, M. (2003). Patient navigation in cancer care: Program delivery and research in British Columbia. Canadian Oncology Nursing Journal, 13(3), 193.

Dorr, D.A., Wilcox, A., Burns, L., Brunker, C.P., Narus, S.P., \& Clayton, P.D. (2006). Implementing a multidisease chronic care model in primary care using people and technology. Disease Management, 9(1), 1-15.

Epping-Jordan, J.E., Pruitt, S.D., Bengoa, R., \& Wagner, E.H. (2004). Improving the quality of health care for chronic conditions. Quality \& Safety in Health Care, 13, 299-305.

Epstein, R.M., \& Street, R.L. (2007). Patient-centered communication in cancer care: Promoting healing and reducing suffering. Bethesda, MD: NIH Publication No. 076225.

Fillion, L., de Serres, M., Lapointe-Goupil, R., Bairati, I., Gagnon, P., Deschamps, M., et al. (2006). Implementing the role of patientnavigator nurse at a university hospital centre. Canadian Oncology Nursing Journal, 16(1), 11-17.

Fischer, S.M., Sauaia, A., \& Kutner, J.S. (2007). Patient navigation: A culturally competent strategy to address disparities in palliative care. Journal of Palliative Medicine, 10(5), 1023-1028.

Fitch, M.I. (2008). Supportive care framework. Canadian Oncology Nursing Journal, 18(1), 6-14.

Fitch, M.I., Cook, S., \& Plante, A. (2008). Cancer patient navigation workshops: Improving access to cancer care. A report by the Cancer Journey Action Group. Toronto, ON: Canadian Partnership Against Cancer.

Fitch, M.I., Porter, H.B., \& Page, B.D. (Eds.). (2008). Supportive care framework: A foundation for person-centred care. Pembroke, ON: Pappin Communications.

Fleissig, A., Jenkins, V., Catt, S., \& Fallowfield, L. (2006). Multidisciplinary teams in cancer care: Are they effective in the UK? Lancet Oncology, 7, 935-43, 7, 935-943.

Freeman, H.P. (2004). Poverty, culture, and social injustice: Determinants of cancer disparities. CA: A Cancer Journal for Clinicians, 54, 72-77.
Freund, K.M., Battaglia, T.A., Calhoun, E., Dudley, D.J., Fiscella, K., Paskett, E., et al. (2008). National Cancer Institute patient navigation research program: Methods, protocol, and measures. Cancer, 113(12).

Kitson, A. (2004). Drawing out leadership [editorial]. Journal of Advanced Nursing, 48(3), 211.

McKay, C.A., \& Crippen, L. (2008). Collaboration through clinical integration. Nursing Administration Quarterly, 32(2), 109-116.

McKenna, M.T., \& Zohrabian, A. (2009). U.S. burden of disease: Past, present and future. Annals of Epidemiology, 19, 212-219.

McMurtry, R., \& Bultz, B.D. (2005). Public policy, human consequences: The gap between biomedicine and psychosocial reality. Psycho-Oncology, 14, 697-703.

Mick, J. (2008). Factors affecting the evolution of oncology nursing care. Clinical Journal of Oncology Nursing, 12(2), 307-313.

Murray, S., Silver, I., Patel, D., Dupuis, M., Hayes, S.M., \& Davis, D. (2008). Community group practices in Canada: Are they ready to reform their practice? Journal of Continuing Education in the Health Professions, 28(2), 73-78.

Nguyen, T.-U.N., \& Kagawa-Singer, M. (2008). Overcoming barriers to cancer care through health navigation programs. Seminars in Oncology Nursing, 24(4), 270-278.

Reid Ponte, P., Gross, A.H., Winer, E., Connaughton, M.J., \& Hassinger, J. (2007). Implementing an interdisciplinary governance model in a comprehensive cancer center. Oncology Nursing Forum, 34(3), 611-616.

Schroeder, C.A., Trehearne, B., \& Ward, D. (2000). Expanded role of nursing in managed care. Part II: Impact on outcomes of costs, quality, provider and patient satisfaction. Nursing Economics, 18(2), 71-78.

Schwaderer, K.A., \& Itano, J.K. (2007). Bridging the healthcare divide with patient navigation: Development of a research program to address disparities. Clinical Journal of Oncology Nursing, 11(5), 633-639.

Seek, A.J., \& Hogle, W.P. (2007). Modeling a better way: Navigating the healthcare system for patients with lung cancer. Clinical Journal of Oncology Nursing, 11(1), 81-85.

Skrutkowski, M., Saucier, A., Eades, M., Swidzinski, M., Ritchie, J., Marchionni, C., et al. (2008). Impact of a pivot nurse in oncology on patients with lung or breast cancer: Symptom distress, fatigue, quality of life, and use of healthcare resources. Oncology Nursing Forum, 35(6), 948-954.

Sofaer, S. (2009). Navigating poorly charted territory: Patient dilemmas in health care "nonsystems". Medical Care Research and Review, 66(1), 75S-93S.

Thorne, S.E., Bultz, B.D., Baile, W.F., \& SCRN Communication Team. (2005). Is there a cost to poor communication in cancer care?: A critical review of the literature. Psycho-Oncology, 14, 875-884.

Thorne, S.E., Hislop, T.G., Armstrong, E.A., \& Oglov, V. (2008). Cancer care communication: The power to harm and the power to heal? Patient Education \& Counseling, 71(34-40).

Thorne, S.E., Kuo, M., Armstrong E-A., McPherson, G., Harris, S., \& Hislop, G. (2005). "Being known:” Patient perspectives on human connection in cancer care. Psycho-Oncology, 14, 887-898.

Wells, K.J., Battaglia, T.A., Dudley, D.J., Garcia, R., Greene, A., Calhoun, E., et al. (2008). Patient navigation: State of the art or is it science? Cancer, 113, 1999-2010.

Willard, C., \& Luker, K. (2005). Supportive care in the cancer setting: rhetoric or reality? Palliative Medicine, 19, 328-333.

World Health Organization. (2002). Innovative care for chronic conditions: Building blocks for action. Geneva: World Health Organization (WHO/MNC/CCH/02.01). 